\title{
Long noncoding RNA Z38 promotes cell proliferation and metastasis and inhibits cell apoptosis in human gastric cancer
}

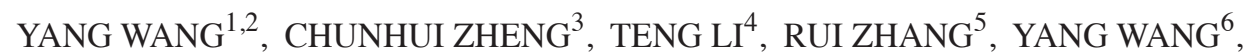 \\ JIAXIN ZHANG ${ }^{6}$, QINGSI HE ${ }^{1}$, ZUOCHENG SUN $^{2}$ and XINSHENG WANG ${ }^{7}$ \\ ${ }^{1}$ Department of General Surgery, Shandong University Qilu Hospital, Jinan, Shandong 250012; \\ Departments of ${ }^{2}$ General Surgery, ${ }^{3}$ Oncology Surgery, ${ }^{4}$ Interventional Radiology, \\ ${ }^{5}$ Gynecology and ${ }^{6}$ Breast Surgery, Weifang People's Hospital, Weifang, Shandong 261041; \\ ${ }^{7}$ Department of General Surgery, Anqiu People's Hospital, Weifang, Shandong 262100, P.R. China
}

Received March 26, 2017; Accepted January 26, 2018

DOI: $10.3892 / \mathrm{ol} .2018 .9343$

\begin{abstract}
Gastric cancer is one of the leading causes of cancer-associated mortality and has a high tendency to metastasize, making it a priority to develop novel diagnostic and treatment methods at the early stages. The present study investigated the role of a newly-discovered long non-coding RNA, Z38, in gastric cancer cell proliferation, metastasis and apoptosis. It was observed that Z38 was upregulated in tissues from patients with gastric cancer as well as in cultured gastric cancer cells. Knockdown of Z38 decreased the cell proliferative rate, as evidenced by colony formation assays and cell proliferation assays. In addition, Transwell assays and wound-healing assays demonstrated that depletion of Z38 significantly inhibited cell migration and invasion in AGS and MKN74 cells. Furthermore, a cell apoptosis assay and measurement of relative activities of related caspases revealed that depletion of Z38 increased cell apoptosis by promoting the activities of caspase- 3 and caspase- 9 , but not that of caspase- 8 . Finally, western blot analysis further demonstrated the role of Z38 in the apoptosis of AGS and MKN74 cells. These results suggested that Z38 promotes cell proliferation and metastasis, and inhibits cell apoptosis in gastric cancer. Z38 may represent a novel therapeutic target for the treatment of gastric cancer in clinic.
\end{abstract}

\section{Introduction}

Gastric cancer is among the most common types of cancer worldwide with an increasing incidence rate each year.

Correspondence to: Dr Qingsi He, Department of General Surgery, Shandong University Qilu Hospital, 107 West Culture Road, Jinan, Shandong 250012, P.R. China

E-mail: yll11444@163.com

Dr Zuocheng Sun, Department of General Surgery, Weifang People's Hospital, 151 Guangwen Street, Weifang, Shandong 261041, P.R. China E-mail: zuocheng1966sun@gmail.com

Key words: Z38, gastric cancer, proliferation, metastasis, apoptosis
Approximately 650,000 patients succumb to gastric cancer, making its mortality rate subsequent to that of lung cancer (1). Various factors attribute to the occurrence of gastric cancer, including the genetic background of patients and environmental factors (2). Although substantial efforts have been made in the diagnosis and treatment of patients with gastric cancer, little breakthrough has been made in previous decades due to its high tendency for metastasis. Numerous patients are diagnosed at such an advanced stage that even combined chemotherapy or radiotherapy fail to yield a satisfactory outcome (3). Therefore, there is an urgency to develop novel therapeutic targets for the treatment of gastric cancer, particularly for those tolerant to traditional therapies.

It is known that only $2 \%$ of the mammalian genome is able to be translated into protein; however, $>85 \%$ of the genome exhibits the potential to be transcribed into RNA, while the majority of RNAs serve roles in regulation $(4,5)$. Notably, long non-coding RNAs (lncRNAs) are among the regulatory RNAs, which have a length of $>200$ nucleotides (6). IncRNAs have been reported to interact with DNAs, RNAs and proteins, and are involved in the processes of DNA transcription, the cell cycle, apoptosis and autophagy (7). Multiple lncRNAs have been demonstrated to participate in the tumorigenesis of gastric cancer. IncRNA AGAP2-AS1 was revealed to be activated by SP1, and promoted cell proliferation and metastasis in patients with gastric cancer (8). IncRNA PVT1 functions as a competing endogenous RNA via sponging microRNA 186 in gastric cancer (9).

Z38 was a newly-discovered lncRNA by Deng et al in 2016 (10). Z38 was demonstrated to be a protein coding isoform of claudin domain containing 1 mRNA, which belongs to the claudin family, a family that contains $>26$ members and is characterized by a common motif in the para-cellular loop (11). Z38 was demonstrated to be an lncRNA by in vitro translation experiments and was markedly upregulated in human breast cancer (12). Knockdown of Z38 in breast cancer cells inhibited cell proliferation and metastasis (10). However, the detailed mechanism of the inhibitory roles of Z38 in breast cancer remains unknown. Furthermore, the role of $\mathrm{Z} 38$ in other malignancies requires further investigation. 
In the present study, the relative transcript levels of Z38 were examined in patients with gastric cancer and in cultured cells. The roles of Z38 in cell proliferation and metastasis were examined with cell viability assays, colony formation assays and Transwell assays, as well as wound-healing assays. A preliminary study focusing on the effects of $\mathrm{Z} 38$ on cell apoptosis was also included. The results of the present study indicated that Z38 may act as a potential therapeutic target for the treatment of gastric cancer.

\section{Materials and methods}

Human samples. Gastric cancer tissues and matched adjacent non-cancerous tissues from 100 patients (age range: 35-75 years, average age: 62 years, males: 37, females: 63) who were admitted to the Department of General Surgery, Weifang People's Hospital (Weifang, China) between April 2014 and May 2016, were collected following surgical resection and were immediately frozen in liquid nitrogen. Clinical characteristics of these patients, including age, sex, presenting symptoms and TNM stage were also assessed (13). Written informed consent was obtained from each patient and the present study was approved by the Ethics Committee of Weifang People's Hospital.

Cell culture and antibodies. The human gastric cancer KATO III, SGC-7901 and AGS cell lines, as well as the 293T cell line as a control, were purchased from the cell bank of the Chinese Academy of Sciences (Shanghai, China). The human gastric cancer MKN45 and MKN74 cell lines were purchased from American Type Culture Collection (Manassas, VA, USA). All cell lines were cultured in Dulbecco's modified Eagle's medium (DMEM; Gibco; Thermo Fisher Scientific, Inc., Waltham, MA, USA), supplemented with $10 \%$ fetal bovine serum (FBS; Gibco; Thermo Fisher Scientific, Inc.). Primary antibodies against caspase- 3 and caspase-9 were purchased from Cell Signaling Technology, Inc. (Danvers, MA, USA). The primary antibody against GAPDH and the horseradish peroxidase-conjugated secondary antibodies were purchased from Santa Cruz Biotechnology, Inc. (Dallas, TX, USA).

Total RNA extraction and reverse transcription-quantitative polymerase chain reaction $(R T-q P C R)$. Total RNA was extracted from human tissues and cultured gastric cancer cells using TRIzol ${ }^{\circledR}$ reagent (Takara Biotechnology Co., Ltd., Dalian, China), according to the manufacturer's protocols. The RNA quality and concentration were determined by collecting the absorbance with the Nanodrop 2000 spectrophotometer (Thermo Fisher Scientific, Inc.). Reverse transcription (RT) of first-strand cDNAs $(1 \mu \mathrm{g})$ was performed using PrimeScript RT Master mix (Takara Biotechnology Co., Ltd.), according to the manufacturer's protocol. All PCR amplifications were performed in an ABI PRISM 7900 Real-Time system (Applied Biosystems; Thermo Fisher Scientific, Inc.) with the SYBR ${ }^{\circledR}$ Premix Ex Taq ${ }^{\mathrm{TM}}$ kit (Takara Biotechnology Co., Ltd.). The thermocycling conditions were as follows: Initial denaturation at $95^{\circ} \mathrm{C}$ for $5 \mathrm{~min}$, followed by 45 repeats of a three-step cycling program consisting of $10 \mathrm{sec}$ at $95^{\circ} \mathrm{C}$ (denaturation), $10 \mathrm{sec}$ at $60^{\circ} \mathrm{C}$ (primer annealing) and $10 \mathrm{sec}$ at $72^{\circ} \mathrm{C}$ (elongation), and a final extension step for $10 \mathrm{~min}$ at $72^{\circ} \mathrm{C}$. The primer sequences used for qPCR are listed in Table I and GAPDH was used as the internal control. Primers were synthesized by Shanghai Shenggong Biology Engineering Technology Service, Ltd. (Shanghai, China). All quantitative data were normalized to GAPDH using the $2^{-\triangle \Delta \mathrm{Cq}}$ method (14).

Small interfering RNA (siRNA) interference. For knockdown of Z38, specific siRNAs (siZ38-1 and siZ38-2, 1uM) were designed and synthesized by Santa Cruz Biotechnology, Inc. and diluted to a final concentration of $20 \mathrm{mM}$. The transfection assay was performed using Lipofectamine ${ }^{\circledR} 2000$ transfection reagent (Invitrogen; Thermo Fisher Scientific, Inc.), according to the manufacturer's protocols. At $6 \mathrm{~h}$ after transfection, the medium was replaced with fresh Dulbecco's modified Eagle's medium (DMEM) containing 10\% fetal bovine serum. The cells were subject to subsequent analysis $72 \mathrm{~h}$ later.

Colony formation assay. AGS and MKN74 cells were seeded onto 12-well plates $24 \mathrm{~h}$ prior to transfection, following which specific siRNA against Z38 was transfected. Subsequently, a total of 500 cells were seeded onto a 6 -well plate in each treatment group. The plates were incubated at $37^{\circ} \mathrm{C}$ for 2 weeks without changing the culture medium and mixed with methanol at room temperature for $15 \mathrm{~min}$. Finally, the colonies were stained with $1 \%$ crystal violet for $10 \mathrm{~min}$ at room temperature and images were captured (Leica DM IRB; Leica Microsystems $\mathrm{GmbH}$, Wetzlar, Germany) in 5 random fields of view. The whole plates were counted under a Nikon light microscope (x200 magnification) and were statically analyzed.

Cell viability assay. AGS and MKN74 cells were seeded onto 96-well plates (3,000 cells/well) and were cultured overnight. Cells were subsequently transfected with siZ38-1 or siz38-2 $(10 \mu \mathrm{M})$, followed by incubation in DMEM for another $72 \mathrm{~h}$. Cell viabilities were determined for 5 consecutive days using the MTT assay. For this, $2 \mathrm{mg} / \mathrm{ml}$ MTT solution was added to each well, followed by incubation for $4 \mathrm{~h}$ at $37^{\circ} \mathrm{C}$. Subsequently, the medium was removed and $200 \mu \mathrm{l}$ dimethyl sulfoxide was added to dissolve the purple formazan. The plate was agitated for $5 \mathrm{~min}$ at room temperature and the optical density was subsequently determined at $570 \mathrm{~nm}$ using a spectrophotometer.

Transwell assays. AGS and MKN74 cells were cultured in 24-well plates and transfected with specific Z38 siRNA or control siRNA, with transfection protocols as stated previously. At 48 h post-transfection, cells were harvested and single-cell suspensions in serum-free DMEM were prepared, of which $150 \mu \mathrm{l}\left(3 \times 10^{4}\right.$ cells $)$ was seeded into the upper chamber of an 8-mm Transwell plate (Corning Life Sciences, Corning, NY, USA). The lower chamber was filled with $600 \mu 1$ DMEM, supplemented with $10 \%$ FBS. For the invasion assay, the membrane was coated with Matrigel (Beyotime Institute of Biotechnology) $6 \mathrm{~h}$ prior to seeding. Following incubation at $37^{\circ} \mathrm{C}$ for $12 \mathrm{~h}$, cells were fixed with ice-cold methanol for $20 \mathrm{~min}$ and stained with $0.1 \%$ crystal violet for $5 \mathrm{~min}$ at room temperature. Images were captured under an inverted light microscope at a magnification of x200.

Wound healing assay. AGS and MKN74 cells were seeded onto 6 -well plates $\left(\sim 5 \times 10^{5}\right.$ cells/well) and transfected with 
Table I. Primers sequences used in reverse transcriptionquantitative polymerase chain reaction.

\begin{tabular}{lc}
\hline Gene & \multicolumn{1}{c}{ Primer nucleotide sequences } \\
\hline Z38 & \\
Forward & 5'-AGTGGGATTGTGGAGACGGTGT-3' \\
Reverse & 5'-AGGTAAAAGGAACTGGCAACGC-3' \\
GAPDH & \\
Forward & 5'-GTGGACATCCGCAAAGAC-3' \\
Reverse & 5'-AAAGGGTGTAACGCAACTA-3' \\
\hline
\end{tabular}

siZ38 or control siRNA. A sterile $10 \mu \mathrm{l}$ pipette tip was used to scrape across the center of each well at $48 \mathrm{~h}$ post-transfection three times and immediately cultured with serum-free medium (Gibco; Thermo Fisher Scientific, Inc.). Cells were allowed to migrate for $12 \mathrm{~h}$, following which scratches were observed and images. Subsequently, cells were rinsed with phosphate-buffered saline were captured for each group using a light microscope (x200 magnification). Each assay was performed in triplicate and repeated at least three times.

Flow cytometric analysis of cell apoptosis. The Annexin $\mathrm{V} /$ propidium iodide (PI) assay was performed according to the manufacturer's protocols (Invitrogen; Thermo Fisher Scientific, Inc.). In brief, AGS and MKN74 cells were plated onto 6-well plates and transfected with control or specific siRNA against Z38. Subsequently, cells were washed with pre-cold phosphate-buffered saline, trypsinized and re-suspended in $100 \mu \mathrm{l}$ binding buffer with $2.5 \mu \mathrm{l}$ fluorescein isothiocyanate-conjugated Annexin-V and $1 \mu \mathrm{l} \mathrm{PI}(100 \mu \mathrm{g} / \mathrm{ml})$. Cells were subsequently incubated at room temperature for $15 \mathrm{~min}$ in the dark. A total of $>10,000$ cells were collected and calculated using a flow cytometer (BD Biosciences, Franklin Lakes, NJ, USA) with FolwJo 7.6.1 software (FlowJo LLC, Ashland, OR, USA).

Determination of caspase activities. The activities of caspase-3, caspase- 8 and caspase- 9 were determined using caspase activity kits (Beyotime Institute of Biotechnology), according to the manufacturer's protocols. In brief, cells were transfected with siRNAs for $72 \mathrm{~h}$. Subsequently, cell lysates were collected by low speed centrifugation $(860 \mathrm{x} \mathrm{g}$ for $5 \mathrm{~min}$ at $\left.4^{\circ} \mathrm{C}\right)$. An equal amount of protein $(10 \mu \mathrm{l})$ from each sample were added to 96-well plates and mixed with an aliquot of $80 \mu 1$ reaction buffer (Beyotime Institute of Biotechnology) supplied with caspase substrates $(2 \mathrm{mM})$. Following incubation at $37^{\circ} \mathrm{C}$ for $4 \mathrm{~h}$, caspase activities were determined using a TECAN reader at an absorbance of $450 \mathrm{~nm}$.

Western blot analysis. Total protein was extracted from cultured cells. Cell lines were permitted to grow until they reached $95 \%$ confluence. Following two washes with phosphate-buffered saline, cells were lysed with a general lysis buffer (NP40; Beyotime Institute of Biotechnology) to generate the total protein lysate. The protein was quantified with a Pierce BCA Protein Assay kit (Thermo Fisher Scientific, Inc.). Protein was then subjected to $10 \%$ SDS-PAGE by loading equal amounts of whole protein $(50 \mu \mathrm{g})$ per lane. PVDF membranes were blocked with $5 \%$ milk in TBST at room temperature for $1 \mathrm{~h}$ and then incubated with the following antibodies at $4{ }^{\circ} \mathrm{C}$ for overnight: Anti-caspase-3 (cat. no. 9662; dilution, 1:1,000), anti-caspase-9 (cat. no. 9508; dilution, 1:1,000), anti-GAPDH (cat. no. sc-47724; dilution, 1:2000), prior to being incubated with a horseradish peroxidase-conjugated secondary antibody (Santa Cruz Biotech., Santa Cruz, USA, dilution, 1:5,000 at room temperature for $1 \mathrm{~h}$ ). GAPDH was synchronously detected as a loading control. Immunoreactivity was determined using enhanced chemiluminescence autoradiography (Thermo Fisher Scientific., Inc.). Image J 2x software (National Institutes of Health, Bethesda, MD, USA) was used to quantify the western blotting data. Each experiment was repeated at least three times.

Statistical analysis. Data are expressed as the mean \pm standard deviation. A two-tailed Student's t-test was used to compare the means of two groups, while one-way analysis of variance was used for comparisons among multiple groups ( $\geq 3$ groups), followed by a least significant difference post hoc test. $\mathrm{P}<0.05$ was considered to indicate a statistically significant difference. All experiments were repeated at least three times unless otherwise stated.

\section{Results}

IncRNA Z38 was overexpressed in patients with gastric cancer and in gastric cancer cells. In order to investigate the role of Z38 in gastric cancer, the relative transcript level of Z38 in 100 clinical gastric cancer tissues was examined. As demonstrated in Fig. 1A, the relative expression of Z38 was significantly increased in the clinical gastric cancer tissues compared with expression in the adjacent non-cancerous tissues $(\mathrm{P}<0.0001)$. Clinical characteristics of these patients were also assessed. It is demonstrated in Table II that the expression of Z38 was associated with tumor size, lymph node metastasis, distant metastasis and Tumor-Node-Metastasis (TNM) staging (13), but was not associated with age, sex or presenting symptoms. Subsequently, the expression of Z38 in gastric cancer cells was examined, using 293T cells as a control. Compared with the control cells, all the gastric cancer cells exhibited higher expression of Z38 (Fig. 1B). Of note, it was verified that AGS and MKN74, the two most invasive cell lines, exhibited the highest expression of Z38, indicating the potential role of Z38 in cell metastasis. These data suggested that the transcript level of Z38 was upregulated in human gastric cancer.

Knockdown of Z38 in gastric cancer inhibited cell proliferation in vitro. Next, two specific siRNAs against Z38, namely siZ38-1 and siZ38-2, were designed. Subsequently, these two siRNAs were transfected into AGS and MKN74 cells. It was revealed that the relative transcript level of Z38 was significantly decreased by siz38-1, but not siz38-2 (Fig. 2A); therefore, only siz38-1 was included in the subsequent analysis. Colony formation and cell proliferation assays were performed to investigate the role of Z38 in cell proliferation. Approximately 220 colonies were formed in control and siNC-treated AGS cells, while only 150 colonies were observed 

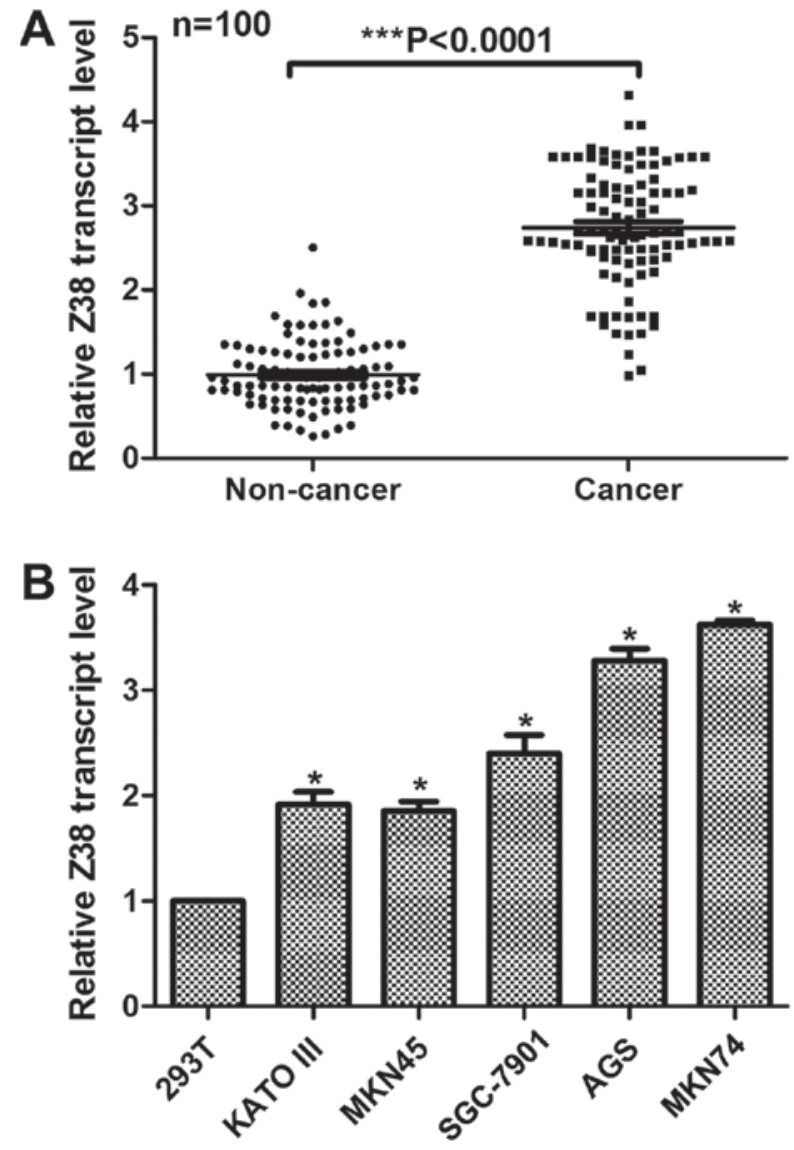

Figure 1. Long non-coding RNA Z38 was overexpressed in human gastric cancer tissues and cells. (A) A total of 100 patients with gastric cancer were included and the expression of Z38 in the tumor tissues and their adjacent non-cancerous tissues was investigated. ${ }^{* * *} \mathrm{P}<0.0001$, Cancer vs. Non-cancer. (B) Five gastric cancer cell lines KATO III, MKN45, SGC-7901, AGS and MKN74 and a control cell line 293T were involved to investigate the expression of $\mathrm{Z} 38$ using reverse transcription-quantitative polymerase chain reaction analysis. ${ }^{*} \mathrm{P}<0.05$ vs. $293 \mathrm{~T}$ cells.

in siZ38-transfected cells (Fig. 2B). A similar phenomenon was also observed in MKN74 cells. In the cell proliferation assays, no significant difference was observed among the three groups in AGS or MKN74 cells in the first three days; however, on the fourth day, the proliferation rate was inhibited by 25 and $20 \%$ in AGS and MKN74 cells, respectively (Fig. 2C and D). Furthermore, the inhibitory effects were even pervasive on the fifth day in the two cell lines. These results revealed that knockdown of Z38 inhibited cell proliferation in the human gastric cancer AGS and MKN74 cell lines.

Depletion of Z38 in human gastric cancer cells suppressed cell metastasis in vitro. In order to further investigate the role of Z38, Transwell and wound-healing assays were performed. It was revealed that $250 \mathrm{AGS}$ and $220 \mathrm{MKN} 74$ cells migrated through the membrane in control and siNC-treated groups, while only 120 AGS and 125 MKN74 cells were observed on the lower surface of the membrane in the migration assay (Fig. 3A). Similarly, the invasive abilities of AGS and MKN74 cells were also suppressed upon siZ38 transfection (Fig. 3B). Furthermore, the wound-healing assay also revealed that the wound closure area was decreased upon siZ38 transfection in the two cell lines (Fig. 3C and D). All these data suggested that knockdown of Z38 in human gastric cancer cells suppressed cell metastasis in vitro.

Knockdown of Z38 in gastric cancer cell lines promoted cell apoptosis in vitro. Increased cell proliferation rate, cell metastasis potential and inhibited cell apoptotic capacity were the main manifestations of the majority of malignancies (15); therefore, the present study also investigated the effects of Z38 on cell apoptosis. As demonstrated in Fig. 4A, transfection with siZ38 increased the cell apoptotic rate by 8 and $7 \%$ in AGS and MKN74 cells, respectively. Furthermore, the relative caspase activities were also determined. The relative activities of caspase-3 (Fig. 4B) and caspase-9 (Fig. 4D) were increased $\sim 2$-fold upon siZ38 transfection compared with the control cells, while the activity of caspase- 8 remained stable (Fig. 4C), indicating that the role of $\mathrm{Z} 38$ was associated with the intrinsic pathway of apoptosis. Subsequently, western blot analysis was performed and it was revealed that in AGS and MKN74 cells, knockdown of Z38 increased the protein levels of caspase-3 and caspase-9 (Fig. 5), which was consistent with the results demonstrated in Fig. 4. These results suggested that depletion of Z38 in AGS and MKN74 cells increased cell apoptosis by promoting the activities of caspase- 3 and caspase- 9 .

\section{Discussion}

Gastric cancer is the fourth most common type of cancer among males and the sixth among females (16). Furthermore, it is the second cause of cancer-associated mortality worldwide (17). In China, gastric cancer is the third most common cause of cancer-associated mortality (18). In the year 2002, the age standardized incidence rate was 22.0/100,000 males and $10.4 / 100,000$ females and the mortality rate was $16.3 / 100,000$ males and 7.9/100,000 females, according to the global estimation-GLOBOCAN 2002 (19). The property of easy distant metastasis makes current therapeutics unable to treat gastric cancer in all patients. Therefore, it is a priority to develop novel therapeutic targets for the clinical treatment of gastric cancer.

Aberrant expression of certain regulatory RNAs markedly influences cancer origination and progression (20). Therefore, investigating the role of different regulatory RNAs in human tumorigenesis has attracted attention worldwide. The present study examined the expression of lncRNA Z38 in clinical gastric cancer tissues and cultured gastric cancer cells. Of note, KATO III and MKN45 are two cell lines that are poorly differentiated, while AGS and MKN74 are highly differentiated and the SGC-7901 cell line is moderately differentiated. Notably, the relative transcript level of Z38 was highest in AGS and MKN74 cells, indicating the potential capacity of Z38 involvement in cell metastasis. One of the limitations of the present study was that only $293 \mathrm{~T}$ cells were included as a control cell line, but not normal gastric cells, as $293 \mathrm{~T}$ cells are widely used as control and tool cells in studies on gene expression in healthy cells and cancer cells (21-23). However, at the time of the present study, there was no access to normal gastric cells and therefore, $293 \mathrm{~T}$ cells were included as the control cell line. In addition, expression of $\mathrm{Z} 38$ was associated with gastric cancer aggressive parameters, including tumor size 
Table II. Association between Z38 and clinical variables among 100 gastric cancer patients.

\begin{tabular}{|c|c|c|c|c|}
\hline \multirow[b]{2}{*}{ Variable } & \multirow[b]{2}{*}{ No. } & \multicolumn{2}{|c|}{ Expression of Z38 } & \multirow[b]{2}{*}{ P-value } \\
\hline & & Low $(n=40)$ & $\operatorname{High}(\mathrm{n}=60)$ & \\
\hline Age, years & & & & 0.526 \\
\hline$<40$ & 18 & 7 & 11 & \\
\hline $40-50$ & 28 & 16 & 12 & \\
\hline$>50$ & 54 & 27 & 27 & \\
\hline Sex & & & & 0.094 \\
\hline Male & 62 & 29 & 33 & \\
\hline Female & 38 & 11 & 27 & \\
\hline \multicolumn{5}{|l|}{ Presenting symptoms } \\
\hline Painless lump & 46 & 21 & 25 & 0.159 \\
\hline Painful lump & 48 & 15 & 33 & \\
\hline Atypical symptoms & 6 & 4 & 2 & \\
\hline $\mathrm{T}, \mathrm{cm}$ & & & & $<0.001^{\mathrm{a}}$ \\
\hline $\mathrm{T} 1(\leq 2)$ & 34 & 21 & 13 & \\
\hline $\mathrm{T} 2(>2$ and $<5)$ & 26 & 12 & 14 & \\
\hline $\mathrm{T} 3(\geq 5)$ & 22 & 6 & 16 & \\
\hline T4 (any size with distant metastasis) & 18 & 1 & 17 & \\
\hline $\mathrm{N}$ & & & & $<0.001^{\mathrm{a}}$ \\
\hline N0 & 44 & 28 & 16 & \\
\hline N1 or above & 56 & 12 & 44 & \\
\hline Distant metastasis (M) & & & & $0.023^{\mathrm{a}}$ \\
\hline M0 & 45 & 24 & 21 & \\
\hline M1 & 55 & 16 & 39 & \\
\hline TNM stage & & & & $0.008^{\mathrm{a}}$ \\
\hline $\mathrm{I} / \mathrm{II}$ & 42 & 19 & 23 & \\
\hline III/IV & 58 & 11 & 47 & \\
\hline
\end{tabular}

${ }^{\mathrm{a}} \mathrm{P}<0.05$. T, tumor size; $\mathrm{N}$, lymph node metastasis; M, distant metastasis; TNM, Tumor-Node-Metastasis.

and TNM staging. Therefore, it was hypothesized that Z38 may exert critical roles in cell proliferation and migration. Cell viability, Transwell and wound-healing assays were therefore performed to confirm this hypothesis. The results of the present study supported this aforementioned hypothesis and suggested the oncogenic property of Z38 in gastric cancer.

The induction of apoptosis may be divided into two categories: The intrinsic and extrinsic pathways. The initiation of the intrinsic pathway is associated with the pro-apoptotic factors, B cell lymphoma-associated X protein (Bax) and B cell lymphoma-associated death promoter (Bad), which leads to increased permeability of the mitochondrial membrane, loss of membrane potential and release of cytochrome $c(24,25)$. Cytochrome $c$ binds to apoptotic protease activating factor- 1 and then pro-caspase- 9 to form a protein complex known as apoptosome, the role of which is to cleave pro-caspase to its active form of caspase- 9 and, in turn, to activate caspase-3 (2). The present study investigated the apoptosis rate upon siZ38 transfection and revealed that Z38 may markedly inhibit cell apoptosis in AGS and MKN74 gastric cancer cells. Knockdown of Z38 promoted the relative activities of caspase- 3 and caspase-9, but not that of caspase- 8 , which is involved in the extrinsic pathway of apoptosis. To the best of our knowledge, activation of caspase- 3 requires proteolytic processing of its inactive zymogen into activated p17 and p12 fragments. Therefore, the lower bands of Fig. 5 were also from cleaved-caspase-3, which had a smaller molecular weight (12KD). However, the top band was specific to cleaved-caspase-3, the molecular weight of which was $17 \mathrm{KD}$. At present, the detailed mechanisms that underlie the biological effects of $\mathrm{Z} 38$ remain to be elucidated, and further investigation of the expression of other apoptosis-related proteins, including Bax, Bad and B cell lymphoma 2 , is warranted. For example, the downstream targets of Z38 may be a useful area of further investigation. At present, our group is working on the construction of an expression plasmid of lncRNA Z38, since gain/loss of function experiments are typical protocols for assessing molecular function. The present study represents only a preliminary study reporting the effects of knockdown of Z38 on gastric cancer proliferation and metastasis. Future 
A

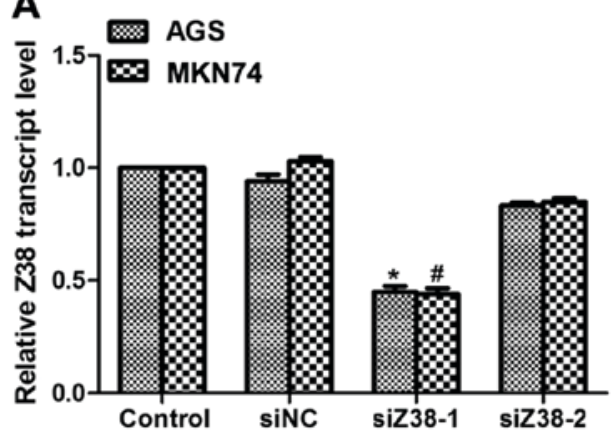

C

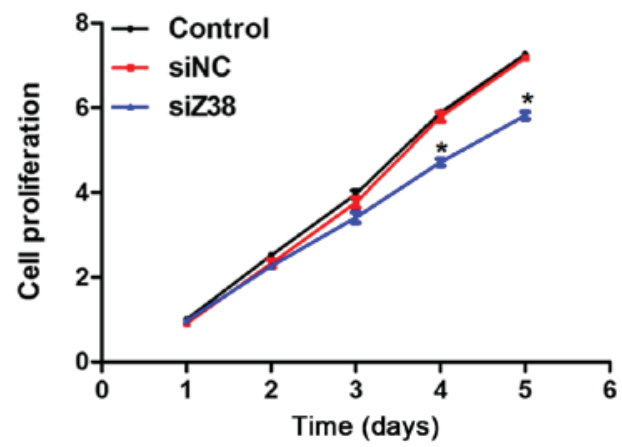

B

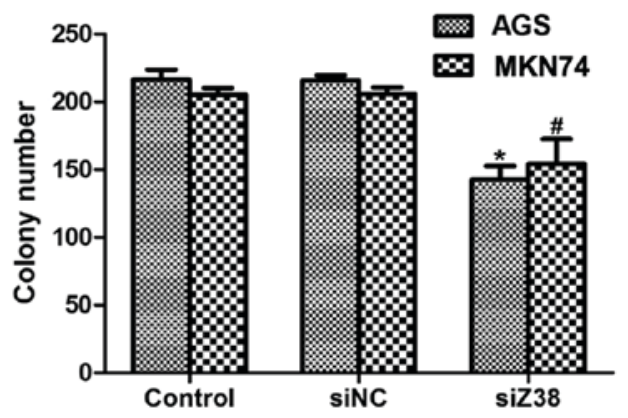

MKN74

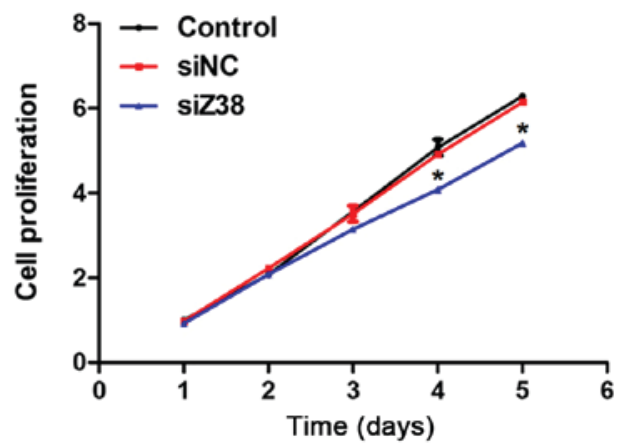

Figure 2. Knockdown of Z38 in AGS and MKN74 cells inhibited cell proliferation. (A) Two specific siRNAs against Z38 were transfected into AGS and MKN74 cells and the relative transcript levels of Z38 were examined. (B) Colony formation assay was performed on siZ38 (short for siZ38-2) transfection in AGS and MKN74 cells. "P<0.05, vs. Control in AGS cells; ${ }^{*} \mathrm{P}<0.05$, vs. Control in MKN74 cells. (C) The cell proliferation rate was investigated when AGS cells were transfected with siZ38 or siNC. (D) The cell proliferation rate was investigated when MKN74 cells were transfected with siZ38 or control siNC. ${ }^{*} \mathrm{P}<0.05$, vs. Control. siRNA, small interfering RNA; siNC, negative control siRNA.
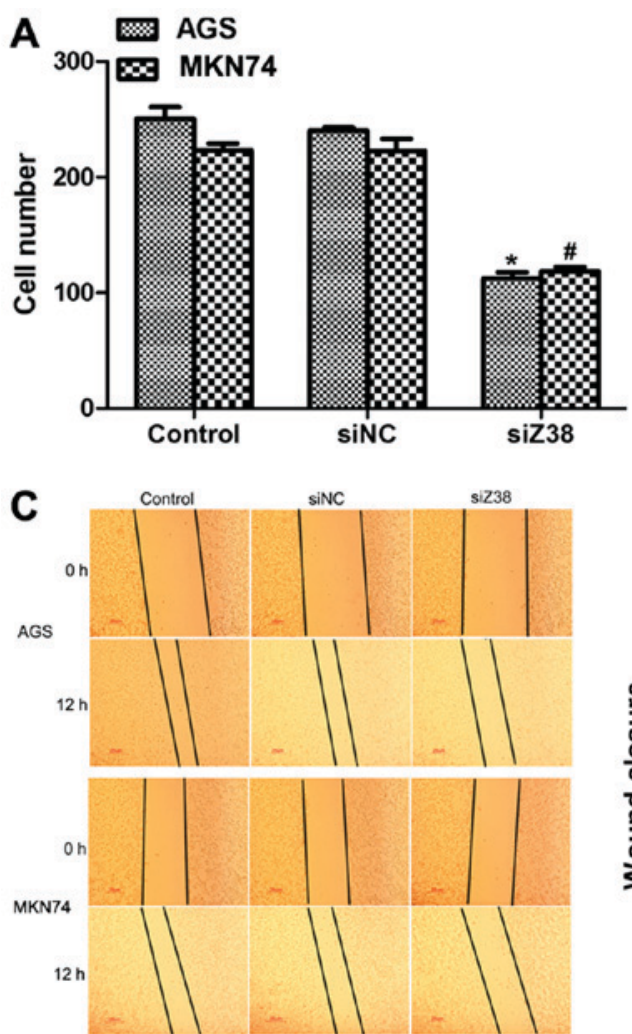

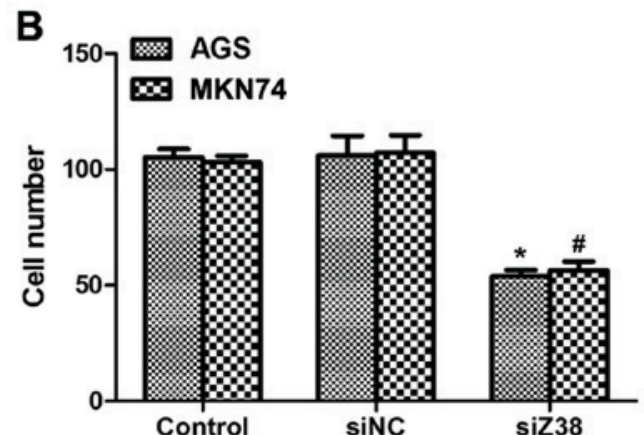

D

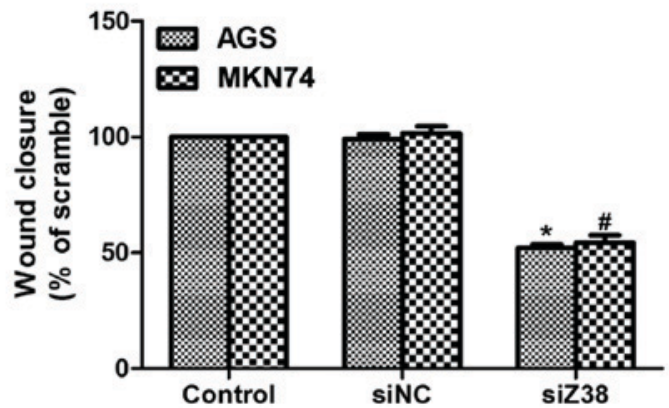

Figure 3. Knockdown of Z38 in AGS and MKN74 cells inhibited cell metastasis. (A) Cell migration assays were performed when AGS and MKN74 cells were transfected with siZ38 for $72 \mathrm{~h}$. (B) Cell invasion assays were performed when AGS and MKN74 cells were treated with siZ38 for $72 \mathrm{~h}$. "P<0.05 vs. Control in AGS cells; " $\mathrm{P}<0.05$ vs. Control in MKN74 cells. (C) Representative images of wound-healing assays. (D) Following random selection of 5 fields for each group of cells, the wound closure rate was quantified in siZ38-transfected AGS and MKN74 cells. ${ }^{*} \mathrm{P}<0.05$ vs. Control in AGS cells; ${ }^{*} \mathrm{P}<0.05$ vs. Control in MKN74 cells. siZ38, Z38 small interfering RNA; siNC, negative control small interfering RNA. 

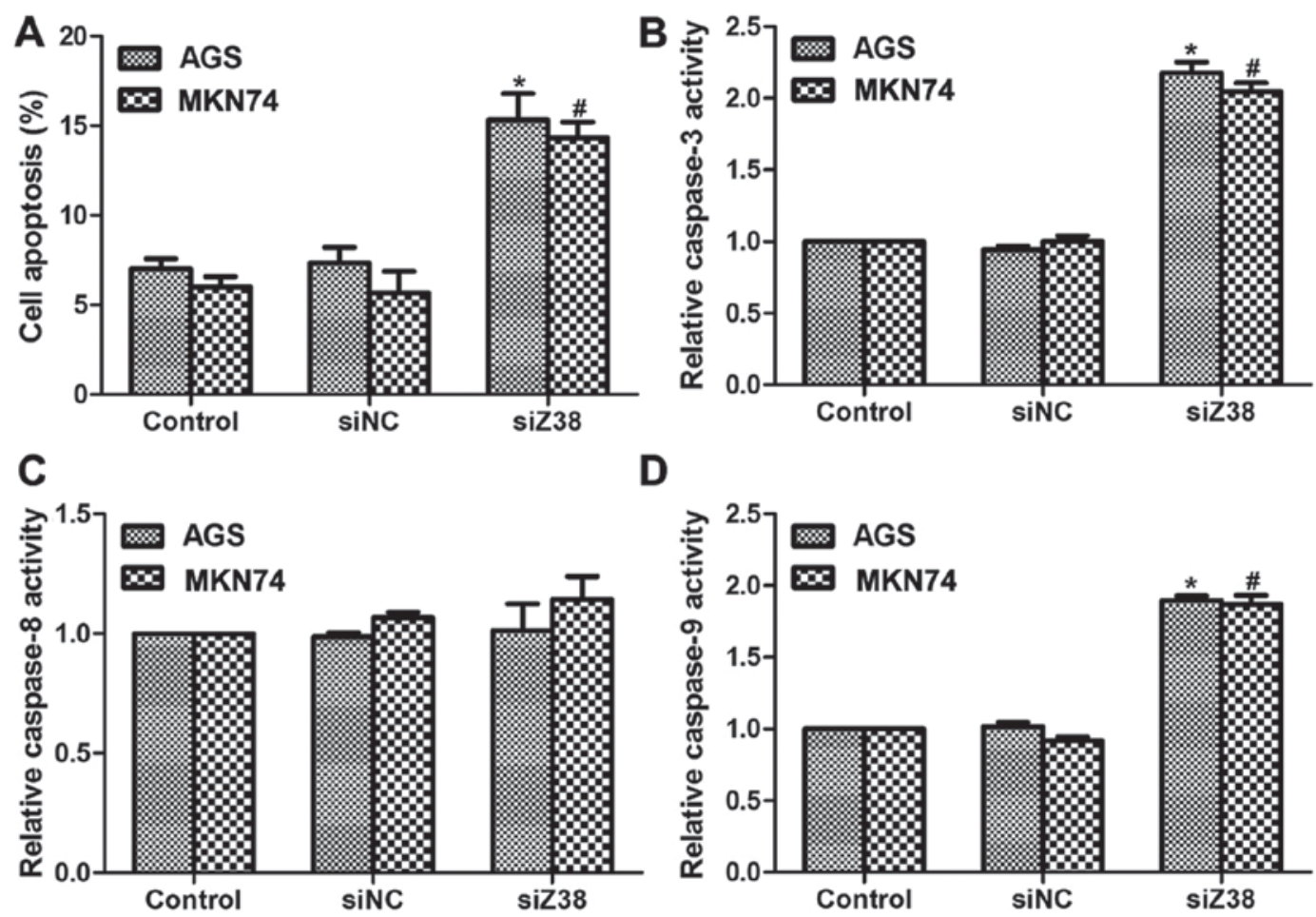

Figure 4. Knockdown of Z38 in AGS and MKN74 cells increased the rate of cell apoptosis. (A) Cell apoptotic rates were examined upon siZ38 transfection in AGS and MKN74 cells. (B) The relative activities of caspase-3 were examined upon siZ38 transfection in AGS and MKN74 cells. (C) The relative activities of caspase-8 were examined upon siZ38 transfection in AGS and MKN74 cells. (D) The relative activities of caspase-9 were examined upon siZ38 transfection in AGS and MKN74 cells. "P $<0.05$ vs. Control in AGS cells; ${ }^{*} \mathrm{P}<0.05$ vs. Control in MKN74 cells. siZ38, Z38 small interfering RNA; siRC, negative control small interfering RNA.

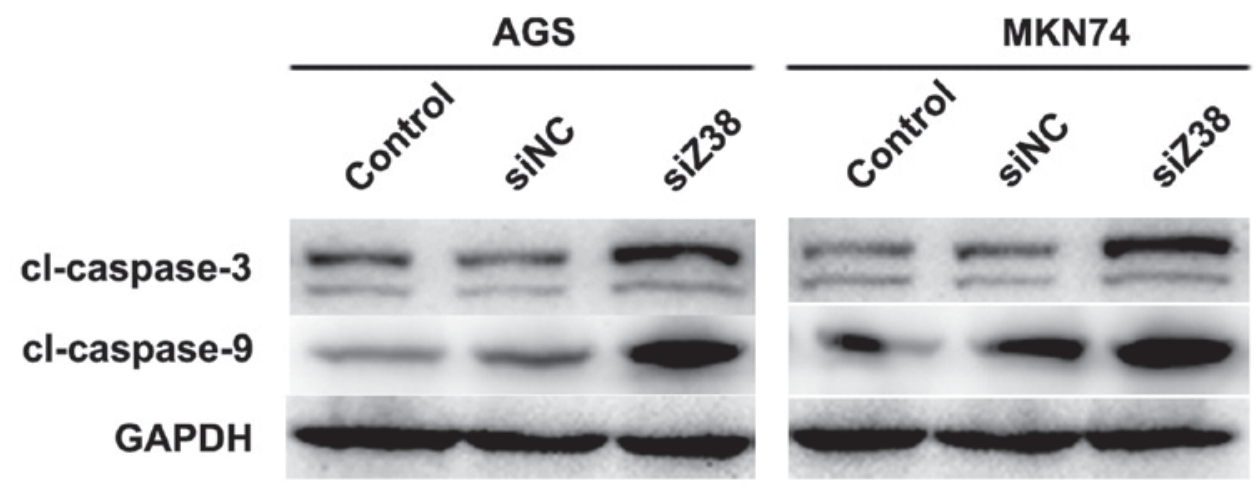

Figure 5. Knockdown of Z38 in AGS and MKN74 cells increased the protein expression levels of caspase-3 and caspase-9. AGS and MNK74 cells were treated with siZ38 for $72 \mathrm{~h}$, and western blot analysis was performed to reveal the protein expression levels of caspase-3 and caspase-9. GAPDH was included as an internal control. Two bands of cl-caspase-3 were detected, but the top bands were specific to cl-caspase-3, the molecular weight of which is $17 \mathrm{KD}$, and the lower one was also from cl-caspase-3, which has a smaller molecular weight (12KD). siZ38, Z38 small interfering RNA; cl-caspase, cleaved-caspase.

studies should aim to perform gain of function experiments and to investigate the detailed molecular mechanisms that contribute to Z38 functions in gastric cancer.

In conclusion, the present study revealed that the expression of Z38 was upregulated in human gastric cancer. Knockdown of Z38 in AGS and MKN74 cells inhibited cell proliferation and metastasis, and promoted cell apoptosis by upregulating the activities of caspase- 3 and caspase-9. The results of the present study indicated the oncogenic potential of Z38 in human gastric cancer and provided evidence that Z38 may serve as a potential therapeutic target for the treatment of gastric cancer.

\section{Acknowledgements}

Not applicable.

\section{Funding}

No funding received.

\section{Availability of data and materials}

The datasets used during the current study are available from the corresponding author on reasonable request. 


\section{Authors' contributions}

YW and CZ performed the experiments. XW and TL analyzed the data. RZ, YW and JZ analysed the data and revised the manuscript and references. $\mathrm{QH}$ and $\mathrm{ZS}$ designed the project, analyzed the data and reviewed the manuscript.

\section{Ethics approval and consent to participate}

Written informed consent was obtained from each patient and the present study was approved by the Ethics Committee of Weifang People's Hospital (Weifang, China).

\section{Consent for publication}

The study participants provided written informed consent for the publication of the data included in the present study.

\section{Competing interests}

The authors declare that they have no competing interests.

\section{References}

1. Shirahata A, Sakata M, Kitamura Y, Sakuraba K, Yokomizo K, Goto T, Mizukami H, Saito M, Ishibashi K, Kigawa G, et al: MACC 1 as a marker for peritoneal-disseminated gastric carcinoma. Anticancer Res 30: 3441-3444, 2010.

2. Li JH, Shen WZ, Gu XQ, Hong WK and Wang ZQ: Prognostic value of EUS combined with MSCT in predicting the recurrence and metastasis of patients with gastric cancer. Jpn J Clin Oncol 47: 487-493, 2017.

3. Bai T, Yokobori T, Altan B, Ide M, Mochiki E, Yanai M, Kimura A, Kogure N, Yanoma T, Suzuki M, et al: High STMN1 level is associated with chemo-resistance and poor prognosis in gastric cancer patients. Br J Cancer 116: 1177-1185, 2017.

4. Sun KY, Peng T, Chen Z, Song P and Zhou XH: Long non-coding RNA LOC100129148 functions as an oncogene in human nasopharyngeal carcinoma by targeting miR-539-5p. Aging (Albany NY) 9: 999-1011, 2017.

5. QianY, Liu D, Cao S, Tao Y, Wei D, Li W, Li G, Pan X and Lei D: Upregulation of the long noncoding RNA UCA1 affects the proliferation, invasion, and survival of hypopharyngeal carcinoma. Mol Cancer 16: 68, 2017.

6. Zhu M, Liu J, Xiao J, Yang L, Cai M, Shen H, Chen X, Ma Y, $\mathrm{Hu} \mathrm{S}$, Wang Z, et al: Lnc-mg is a long non-coding RNA that promotes myogenesis. Nat Commun 8: 14718, 2017.

7. Palmieri G, Paliogiannis P, Sini MC, Manca A, Palomba G, Doneddu V, Tanda F, Pascale MR and Cossu A: Long non-coding RNA CASC2 in human cancer. Crit Rev Oncol Hematol 111: 31-38, 2017.

8. Qi F, Liu X, Wu H, Yu X, Wei C, Huang X, Ji G, Nie F and Wang K: Long noncoding AGAP2-AS1 is activated by SP1 and promotes cell proliferation and invasion in gastric cancer. J Hematol Oncol 10: 48, 2017.

9. Huang T, Liu HW, Chen JQ, Wang SH, Hao LQ, Liu M and Wang B: The long noncoding RNA PVT1 functions as a competing endogenous RNA by sponging miR-186 in gastric cancer. Biomed Pharmacother 88: 302-308, 2017.
10. Deng R, Liu B, Wang Y, Yan F, Hu S, Wang H, Wang T, Li B, Deng X, Xiang S, et al: High expression of the newly found long noncoding RNA Z38 promotes cell proliferation and oncogenic activity in breast cancer. J Cancer 7: 576-586, 2016.

11. Starovasnik MA, Braisted AC and Wells JA: Structural mimicry of a native protein by a minimized binding domain. Proc Natl Acad Sci USA 94: 10080-10085, 1997.

12. Nie ZL, Wang YS, Mei YP, Lin X, Zhang GX, Sun HL, Wang YL, Xia YX and Wang SK: Prognostic significance of long noncoding RNA Z38 as a candidate biomarker in breast cancer. J Clin Lab Anal 32: e22193, 2018.

13. Guo P, Huang ZL, Yu P and Li K: Trends in cancer mortality in china: An update. Ann Oncol 23: 2755-2762, 2012.

14. Livak KJ and Schmittgen TD: Analysis of relative gene expression data using real-time quantitative PCR and the 2(-Delta Delta C(T)) Method. Methods 25: 402-408, 2001.

15. Ribeiro RX, Nascimento CILL and Silva AMTC: Genotype association gstm1 null and gastric cancer: Evidence-based meta-analysis. Arq Gastroenterol 54: 101-108, 2017.

16. Lopez-Ramirez MA, Lever-Rosas CD, Motta-Ramirez GA, Rebollo-Hurtado V, Guzman-Barcenas J, Fonseca-Morales JV and Carreno-Lomeli MA: Correlation between preoperative tomographic staging and definitive histopathologic results in gastric cancer at the Hospital Central Militar. Rev Gastroenterol Mex 82: 210-216, 2017 (In English, Spanish)

17. Pan Y, Zhou F, He C, Hui L, Huang T and Wei Y: Leptin-leprb expressed in gastric cancer patients and related to cancer-related depression. Biomed Res Int 2017: 6482842, 2017.

18. Kamangar F, Dores GM and Anderson WF: Patterns of cancer incidence, mortality, and prevalence across five continents: Defining priorities to reduce cancer disparities in different geographic regions of the world. J Clin Oncol 24: 2137-2150, 2006.

19. Tian Q, Xiao Y, Wu Y, Liu Y, Song Z, Gao W, Zhang J, Yang J, Zhang Y, Guo T, et al: MicroRNA-33b suppresses the proliferation and metastasis of hepatocellular carcinoma cells through the inhibition of Sal-like protein 4 expression. Int J Mol Med 38: 1587-1595, 2016

20. Derrien T, Johnson R, Bussotti G, Tanzer A, Djebali S, Tilgner H, Guernec G, Martin D, Merkel A, Knowles DG, et al: The gencode v7 catalog of human long noncoding rnas: Analysis of their gene structure, evolution, and expression. Genome Res 22: 1775-1789, 2012.

21. Cai Y, Yi M, Chen D, Liu J, Guleng B, Ren J and Shi H: Trefoil factor family 2 expression inhibits gastric cancer cell growth and invasion in vitro via interactions with the transcription factor $\mathrm{sp} 3$. Int J Mol Med 38: 1474-1480, 2016.

22. Jiang S, Chen R, Yu J, Li N, Ke R, Luo L, Zou J, Zhang J, Zhang K, Lu N and Huang D: Clinical significance and role of LKB1 in gastric cancer. Mol Med Rep 13: 249-256, 2016.

23. Spencer SL and Sorger PK: Measuring and modeling apoptosis in single cells. Cell 144: 926-939, 2011.

24. Chen SD, Wu CL, Hwang WC and Yang DI: More insight into bdnf against neurodegeneration: Anti-apoptosis, anti-oxidation, and suppression of autophagy. Int J Mol Sci 18: E545, 2017.

25. Dejean LM, Martinez-Caballero S and Kinnally KW: Is MAC the knife that cuts cytochrome $\mathrm{c}$ from mitochondria during apoptosis? Cell Death Differ 13: 1387-1395, 2006.

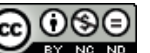

This work is licensed under a Creative Commons Attribution-NonCommercial-NoDerivatives 4.0 International (CC BY-NC-ND 4.0) License. 